\title{
The Upper Critical Field in Disordered Two-Dimensional Superconductors
}

\author{
Robert A Smith ${ }^{1,2}$, Beccy S Handy ${ }^{1}$ and Vinay Ambegaokar ${ }^{2}$ \\ ${ }^{1}$ School of Physics and Astronomy, University of Birmingham, Edgbaston, Birmingham B15 2TT, ENGLAND \\ ${ }^{2}$ Laboratory of Atomic and Solid State Physics, Cornell University, Ithaca, New York 14853, USA
}

\begin{abstract}
We present calculations of the upper critical field $H_{c 2}(T)$ in superconducting films as a function of increasing disorder (as measured by the normal state resistance per square $R_{\square}$ ). In contradiction to previous work, we find that there is no anomalous low temperature positive curvature in $H_{c 2}$ vs $T$ as disorder is increased. We show that the previous prediction of this effect is due to an unjustified analytical approximation of sums occuring in the perturbative calculation. Our treatment includes both a careful analysis of first-order perturbation theory, and a non-perturbative resummation technique. No anomalous curvature is found in either case. We present our results in graphical form.
\end{abstract}

\section{INTRODUCTION}

Increasipg-disorder is known to suppress superconductivity in low-dimensional systems such as thin films and narrow wires 1 . This occurs because the disorder causes electrons to move diffusively rather than ballistically, making them less efficient at screening the Coulomb repulsion between electrons. The increased Coulomb repulsion decreases both the electron-electron attraction needed for superconductivity, and the density of states of electrons available for pairing at the Fermi surface 5 . Typical types of experimental data are: (i) $T_{c}\left(R_{\square}\right)$, the transition temperature as a function of normal state resistance per squared 10; (ii) $\Delta_{0}\left(R_{a}\right)$, the order parameter at zero temperature, as a function of normal state resistance per square11.12; (iii) $H_{0}\left(T, R_{a}\right) \equiv T_{c}\left(R_{a}, H\right)$, the upper critical field as a function of temperature and normal state resistance per squared,4.15; (iv) $T_{c}\left(R_{a}, 1 / \tau_{s}\right)$, transitipn temperature as a function of resistance per square and spin-flip scattering rate in films with magnetic impurities. 13 It is found experimentally that $T_{c}\left(R_{\mathbf{D}}\right)$ curves from a wide variety of materials fit a universal curve with a single fitting parameter, whilst the few experimental measurements of $\Delta_{0}\left(R_{\square}\right)$, seem to have $\Delta_{0}\left(R_{\square}\right) / 2 k_{B} T_{c}\left(R_{\square}\right)$ roughly constant 11 12. This fitting to a single curve, whilst pleasing in showing that the basic ingredients of our theories are correct, does not allow detailed analysis of the theory. Data of types (iii) and (iv) are more promising because there is an additional parameter to vary - the magnetic field in (iii), and spin-flip scattering rate in (iv). To the best of our knowledge, only one experiment of type

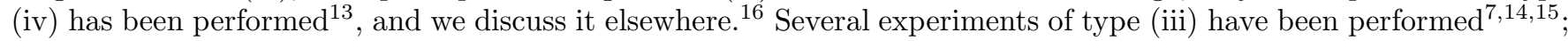
some seem to show a pesitive curvature in $H_{c 2}(T)$ at low temperature as disorder is increased. Moreover this effect is predicted by theory 1218 , and this seems to be another confirmation of the basic theoretical model. However, we need to be careful: positive curvature in $H_{c 2}(T)$ is a ubiquitous feature of exotic superconductors 19 , and occurs in many systems where localization is not believed to be the cause. Indeed any pair-breaking mechanism that varies as a function of magnetic field can lead to such anomalous behaviour in $H_{c 2}$. This means that it is often difficult to distinguish between the various mechanisms that might be present. It is therefore particularly important to be sure of our theory, and in this light we re-examine the predictions of localization theory.

One of the main problems of the localization theory is that even first-order perturbation theory results are hard to obtain correctly. The first-order results are capable of explaining experimental data in the weak disorder regime, but for strepger disorder it is clear that we need something else. As an example consider the prediction for $T_{c}$ suppressionl 1 ,

$$
\ln \left(\frac{T_{c}}{T_{c 0}}\right)=-\frac{1}{3} \frac{R_{\mathrm{a}}}{R_{0}} \ln ^{3}\left(\frac{1}{2 \pi T_{c 0} \tau}\right)
$$

where $T_{c 0}$ is transition temperature for clean system, $R_{0}=2 \pi h / e^{2} \approx 162 k \Omega$, and $\tau$ is the elastic scattering time. This yields an exponential curve for $T_{c}\left(R_{\square}\right)$, which behaves like a straight line for small $R_{\square}$. It is clear that $T_{c}$ deduced from this equation can never go to zero for finite $R_{\square}$, as happens in experiment. A very simple ad hoc way of going beyond simple perturbation theory is to replace $T_{c 0}$ on the right hand side by $T_{c}$, pleading perhaps to self-consistency. If we define $x=\ln \left(T_{c 0} / T_{c}\right), \beta=\ln \left(1 / 2 \pi T_{c 0} \tau\right)$, and $t=R_{\square} / R_{0}$, the new equation has the cubic form

$$
x=\frac{t}{3}(\beta+x)^{3}
$$

and can easily be solved. However a new problem emerges because there are two positive roots for every value of $R_{\mathrm{a}}$. At first we can take the larger of the roots, on physical principles, because it is this root which tends to $T_{c} / T_{c 0}=1$ 
at $R_{\square}=0$. However we eventually come to a re-entrance point beyond which no solutions exist. It is clear that this re-entrance is unphysical, an artefact of our ad hoc extension of perturbation theory. In the case of $T_{c}\left(R_{\square}\right)$ the story has a happy ending in that perturbation theory can be correctly extended by a renormalization group (RG) treatment based on Finkelstein's interacting non-linear sigma model 320 . This leads to the result

$$
\ln \left(\frac{T_{c}}{T_{c 0}}\right)=\frac{1}{|\gamma|}-\frac{1}{2 \sqrt{t}} \ln \left(\frac{1+\sqrt{t} /|\gamma|}{1-\sqrt{t} /|\gamma|}\right)
$$

where $\gamma=-1 / \ln \left(T_{c 0} / 1.13 \tau\right)$. This equation reduces to the first-order result for small $t$ and now $T_{c}$ goes smoothly to zero at $t=\gamma^{2}$. The three curves are plotted for comparison in Fig. (1).

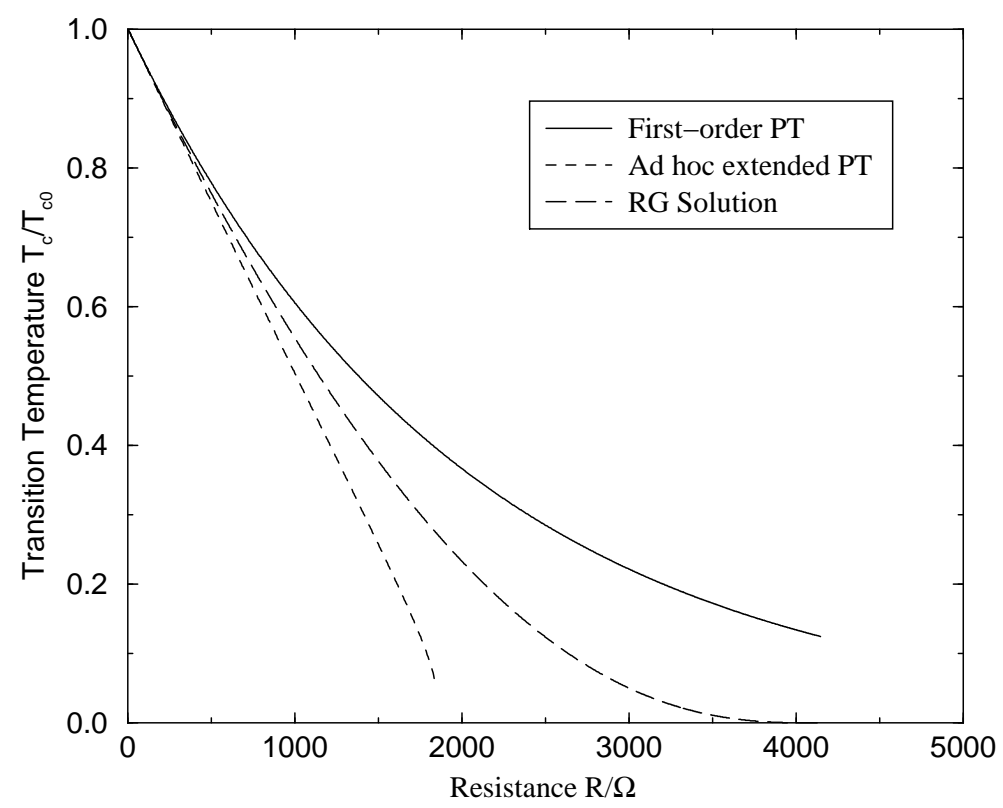

FIG. 1. Theoretical predictions for transition temperature as a function of resistance per square. The solid line is the first-order prediction; the dashed line is the "self-consistent" first-order prediction, which suffers from re-entrance; the long-dashed line is the result obtained from either renormalization group or ladder re-summation approaches.

The reason for discussing $T_{\mathbf{L}}\left(R_{\square}\right)$ in detail above is that the same problem occurs for $H_{c 2}\left(T, R_{\mathbf{\square}}\right) \equiv T_{c}\left(R_{\square}, H\right)$. The standard theory in this case 17 , due to Maekawa, Ebisawa and Fukuyama (MEF) is the equivalent of the ad hoc extension discussed above, and has the form

$$
\begin{aligned}
\ln \left(\frac{T_{c}}{T_{c 0}}\right) & =\psi\left(\frac{1}{2}\right)-\psi\left(\frac{1}{2}+\frac{1}{2 \pi T_{c} \tau_{H}}\left[1-2 t \ln \left(\frac{1}{2 \pi T_{c} \tau}\right)\right]\right)-R_{H F}-R_{V} \\
R_{H F} & =-\frac{1}{2} t \ln ^{2}\left(\frac{1}{2 \pi T_{c} \tau}\right)-t \ln \left(\frac{1}{2 \pi T_{c} \tau}\right)\left[\psi\left(\frac{1}{2}\right)-\psi\left(\frac{1}{2}+\frac{1}{2 \pi T_{c} \tau_{H}}\right)\right] \\
R_{V} & =-\frac{1}{3} t \ln ^{3}\left(\frac{1}{2 \pi T_{c} \tau}\right)-t \ln ^{2}\left(\frac{1}{2 \pi T_{c} \tau}\right)\left[\psi\left(\frac{1}{2}\right)-\psi\left(\frac{1}{2}+\frac{1}{2 \pi T_{c} \tau_{H}}\right)\right]
\end{aligned}
$$

where $1 / \tau_{H}=D e H$. This equation suffers similar re-entrance problems at finite $1 / \tau_{H}$ to those found at $1 / \tau_{H}=0$, when it is just the $T_{c}$ equation. Indeed the $H_{c 2}(T)$ curves can only be plotted down to the value of $T$ at which re-entrance occurs, and at this point the curves appear to have infinite slope. This leads to us asking the question of whether the positive curvature in $H_{c 2}(T)$ is also an artefact of the ad hoc approximation used. What we need to answer this question is the finite magnetic field analogue of the RG result discussed above. However the RG is very difficult, and the answer is not forthcoming from this source. Fortunately Oreg and Finkelstein 21 have recently shown that the RG result can be obtained from a diagrammatic resummation technique. This method has the great advantage of being analytically tractable and easy to use. In this paper we extend this approach to finite magnetic field to see whether we really do expect positive curvature in $H_{c 2}(T)$. Indeed this paper is intended as somewhat of a showcase for this resumpation method, to demonstrate the ease of its extension to a wide variety of problems.

Oreg and Finkel'stein 21 take the Coulomb interaction to be featureless, and we shall follow them. We should therefore explain why it is legitimate to use a featureless interaction rather than the correct screened Coulomb 
interaction. The screened Coulomb interaction has a singularity at low momentum, and one might naively think that this would lead to a strong enhancement of the suppression of transition temperature. However a cancellation-occurs between diagrams 1-4 and diagram 5 of Fig. 2, which effectively removes the low momentum singularityl t. The Coulomb interaction is then effectively featureless, all diagrammatic sums being dominated by large frequency and momentum. It turns out that one gets the same result from doing the perturbation theory correctly with the screened Coulomb interaction as from using a constant interaction of strength $g=N(0) V=1 / 2$ and keeping only diagranes 1-4. This is what we shall do in this paper. We also note that the re-summation method of Oreg and Finkelstein 21 keeps only diagrams 3 and 4, which is again legitimate as their contribution is greater than that from diagrams 1 and 2. However it is not difficult to include these terms in the re-summation, as we will show in section III. The cancellation of low momentum singularities is expected to be a general feature, enforced by gauge invariance, so we are free to ignore them and use a featureless interaction as long as we include the diagrams that give the dominant contribution at large frequency and momentum.

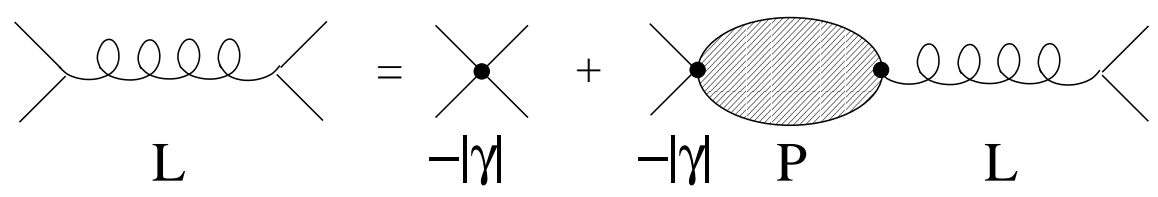

(a)

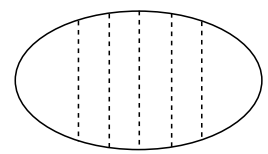

\section{(b)}

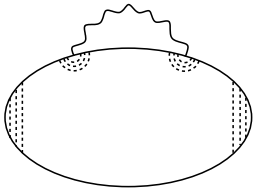

(1a)

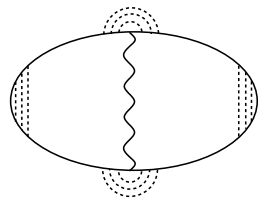

(3a)

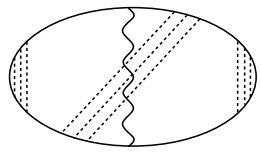

(4b)

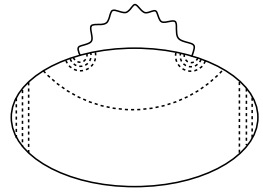

(1b)

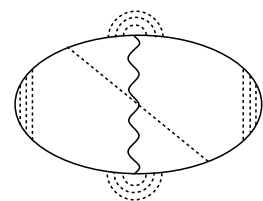

(3b)

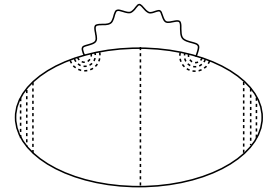

(1c)

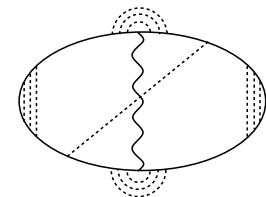

$(3 \mathrm{c})$

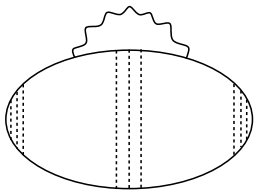

(2)

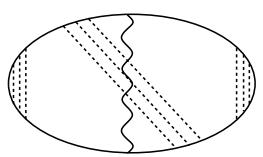

(4a)

(c)

FIG. 2. The diagrams needed to calculate the first-order perturbative correction to the pair-propagator $L(q, 0)$. (a) Definition of pair propagator $L(q, 0)$ in terms of the pair polarization bubble $P(q, 0)$ and the 4-point BCS interaction $-|\gamma|$. (b) Zeroth-order (mean-field) polarization bubble in a dirty superconductor. The solid lines are electron Green functions; the dashed lines are impurity lines. (c) First-order perturbative correction to pair polarization bubble. The wiggly line is the screened Coulomb interaction. 
The outline of the rest of the paper is as follows. In section II we review the first-order perturbation calculation for $H_{c 2}(T)$ assuming a featureless Coulomb interaction. We derive the analytical MEF formula by making an asymptotic approximation to the Matsubara frequency sums which arise in perturbation theory. We make plots of $H_{c 2}(T)$ both by solving the implicit MEF equation, and its equivalent where the Matsubara sums are performed exactly. Surprisingly we find no positive curvature in the latter case. In section III we derive $H_{c 2}(T)$ using the resummation technique both in the form used by Oreg and Finkel'stein, and also in an extended form which includes self-energy diagrams missed in their formalism. Again we find no positive curvature in $H_{c 2}(T)$. In section IV we discuss the experimental situation and draw conclusions.

\section{REVIEW OF FIRST-ORDER PERTURBATION THEORY}

In this section we will carefully review the first-order perturbation theory calculation 17 of $H_{c 2}(T)$. We do this in detail because we will show that accurately performing sums rather than making an analytical approximation to them removes the upward curvature in $H_{c 2}(T)$. We identify $T_{c}$ as the temperature at which the magnetic field dependent pair propagator diverges. To find the latter we calculate the correction to the momentum-dependent pair propagator, $L(q, 0)$, and then make the usual substitution $D q^{2} \rightarrow 2 / \tau_{H}$, where $1 / \tau_{H}=D e H$. The zeroth-order (mean field) pair propagator is given by

$$
L_{0}(q, 0)^{-1}=N(0)\left[\ln \left(\frac{T}{T_{c 0}}\right)+\psi\left(\frac{1}{2}+\frac{D q^{2}}{4 \pi T}\right)-\psi\left(\frac{1}{2}\right)\right]
$$

so that upon substitution $D q^{2} \rightarrow 2 / \tau_{H}$ we get the usual Abrikosov-Gorkov result22 for the pair-breaking effect of the magnetic field

$$
\ln \left(\frac{T_{c}}{T_{c 0}}\right)=\psi\left(\frac{1}{2}\right)-\psi\left(\frac{1}{2}+\frac{1}{2 \pi T_{c} \tau_{H}}\right) .
$$

We will calculate the corrections to the pair polarization bubble, $\delta P(q, 0)$, which will lead to a change in $T_{c}$ given by

$$
\ln \left(\frac{T_{c}}{T_{c 0}}\right)=\psi\left(\frac{1}{2}\right)-\psi\left(\frac{1}{2}+\frac{1}{2 \pi T_{c} \tau_{H}}\right)+\frac{\delta P(q, 0)}{N(0)}
$$

Since we will assume a featureless interaction, we need to eyaluate diagrams 1 to 4 of Fig. 2 . Diagrams 1 and 3 involve the summation of three terms to form a Hikami box 23 , the general form for which is

$$
2 \pi N(0) \tau^{4}\left[-D\left(\Delta_{4}^{1}+2 \Delta_{4}^{2}\right)+\left|\epsilon_{1}\right|+\left|\epsilon_{2}\right|+\left|\epsilon_{3}\right|+\left|\epsilon_{4}\right|\right] \theta\left(-\epsilon_{1} \epsilon_{2}\right) \theta\left(-\epsilon_{2} \epsilon_{3}\right) \theta\left(-\epsilon_{3} \epsilon_{4}\right)
$$

where $\Delta_{4}^{1}=\sum_{i=1}^{4} \mathbf{q}_{i} \cdot \mathbf{q}_{i+1}$ and $\Delta_{4}^{2}=\sum_{i=1}^{2} \mathbf{q}_{i} \cdot \mathbf{q}_{i+2}$, the $\mathbf{q}_{i}$ being the incoming momenta, and the $\epsilon_{i}$ the Matsubara frequencies on the electron Green functions in the box. Using standard diagrammatic rules, and the above result for the Hikami box, we obtain the 4 contributions to the pair polarization bubble

$$
\begin{aligned}
& P_{1}=-4 \pi N(0) V T \sum_{\epsilon_{l}} T \sum_{\epsilon_{n}} \sum_{q^{\prime}} \frac{D q^{2}+D q^{\prime 2}+3\left|\epsilon_{l}\right|+\left|\epsilon_{n}\right|}{\left(D q^{2}+2\left|\epsilon_{l}\right|\right)^{2}\left(D q^{\prime 2}+\left|\epsilon_{l}\right|+\left|\epsilon_{n}\right|\right)^{2}} \theta\left(-\epsilon_{l} \epsilon_{n}\right) \\
& P_{2}=4 \pi N(0) V T \sum_{\epsilon_{l}} T \sum_{\epsilon_{n}} \sum_{q^{\prime}} \frac{1}{\left(D q^{2}+2\left|\epsilon_{l}\right|\right)^{2}\left(D\left(q^{\prime}+q\right)^{2}+\left|\epsilon_{l}\right|+\left|\epsilon_{n}\right|\right)} \theta\left(\epsilon_{l} \epsilon_{n}\right) \\
& P_{3}=-4 \pi N(0) V T \sum_{\epsilon_{l}} T \sum_{\epsilon_{n}} \sum_{q^{\prime}} \frac{D q^{2}+D q^{\prime 2}+\left|\epsilon_{l}\right|+\left|\epsilon_{n}\right|}{\left(D q^{2}+\left|\epsilon_{l}\right|\right)\left(D q^{2}+\left|\epsilon_{n}\right|\right)\left(D q^{\prime 2}+\left|\epsilon_{l}\right|+\left|\epsilon_{n}\right|\right)^{2}} \theta\left(-\epsilon_{l} \epsilon_{n}\right) \\
& P_{4}=-4 \pi N(0) V T \sum_{\epsilon_{l}} T \sum_{\epsilon_{n}} \sum_{q^{\prime}} \frac{1}{\left(D q^{2}+2\left|\epsilon_{l}\right|\right)\left(D q^{2}+\left|\epsilon_{n}\right|\right)\left(D\left(q^{\prime}+q\right)^{2}+\left|\epsilon_{l}\right|+\left|\epsilon_{n}\right|\right)} \theta\left(\epsilon_{l} \epsilon_{n}\right)
\end{aligned}
$$

We note that the relative signs of $\epsilon_{l}$ and $\epsilon_{n}$ are irrelevant in the sum, since the summand depends only upon $\left|\epsilon_{l}\right|$ and $\left|\epsilon_{n}\right|$, and that the featurelessness of the potential allows us to shift the momentum sum in terms 2 and 4 . We find that terms 1 and 2 partially cancel, whilst terms 3 and 4 reinforce, to yield the sum 


$$
P=-\frac{g}{D} T \sum_{\epsilon_{l}>0} T \sum_{\epsilon_{n}>0}\left\{\frac{1}{\left[\epsilon_{l}+1 / \tau_{H}\right]\left[\epsilon_{l}+\epsilon_{n}\right]}+\frac{1}{\left[\epsilon_{l}+1 / \tau_{H}\right]\left[\epsilon_{n}+1 / \tau_{H}\right]}\left[\ln \left(\frac{1}{\left[\epsilon_{l}+\epsilon_{n}\right] \tau}\right)+\frac{1}{\left[\epsilon_{l}+\epsilon_{n}\right] \tau_{H}}\right]\right\}
$$

where $g=N(0) V$ and we have performed the $q^{\prime}$-sum and set $D q^{2} \rightarrow 2 / \tau_{H}$.

We first reproduce MEF's analytic approximation to the sums over Matsubara frequencies. To do this it turns out to be easier to make a choice of relative sign of Matsubara frequencies, $\epsilon_{l} \epsilon_{n}<0$, and to set $\epsilon_{n}=\epsilon_{l}+\omega_{m}$. If $\epsilon_{l}=2 \pi T(l+1 / 2)$ and $\omega_{m}=2 \pi T m$, the sum becomes

$$
\begin{aligned}
P & =-\frac{g}{4 \pi^{2} D} \sum_{m=1}^{M} \sum_{l=0}^{m-1}\left\{\frac{1}{[l+1 / 2+\alpha] m}+\frac{1}{[l+1 / 2+\alpha][m-l-1 / 2+\alpha]}\left[\ln \left(\frac{M}{m}\right)+\frac{\alpha}{m}\right]\right\} \\
& =-\frac{g}{4 \pi^{2} D} \sum_{m=1}^{M}\left[\psi\left(\frac{1}{2}+m+\alpha\right)-\psi\left(\frac{1}{2}+\alpha\right)\right]\left\{\frac{1}{m}+\frac{2}{(m+2 \alpha)}\left[\ln \left(\frac{M}{m}\right)+\frac{\alpha}{m}\right]\right\}
\end{aligned}
$$

where $\alpha=1 / 2 \pi T \tau_{H}, M=1 / 2 \pi T \tau$. If we first evaluate the sum for $\alpha=0$, we see that it will be dominated by large $m$, so we replace the difference of digamma functions by $\ln m$, and the sum over $m$ by an integral to get

$$
P(0)=-\frac{g}{4 \pi^{2} D}\left[\frac{1}{3} \ln ^{3}\left(\frac{1}{2 \pi T_{c} \tau}\right)+\frac{1}{2} \ln ^{2}\left(\frac{1}{2 \pi T_{c} \tau}\right)\right] .
$$

To evaluate the result for finite $\alpha$ we subtract off the $\alpha=0$ result and try to analytically approximate the difference. Again we expect the sum to be dominated by large $m$, so we can ignore the $\alpha$ in $\psi(1 / 2+m+\alpha)$ and $(m+2 \alpha)$, and ignore the term proportional to $\alpha$ as it is less divergent. The difference then has the form

$$
\begin{aligned}
P(\alpha)-P(0) & =-\frac{g}{4 \pi^{2} D} \sum_{m=1}^{M}\left[\psi\left(\frac{1}{2}\right)-\psi\left(\frac{1}{2}+\alpha\right)\right]\left\{\frac{1}{m}+\frac{2}{m} \ln \left(\frac{M}{m}\right)\right\} \\
& =-\frac{g}{4 \pi^{2} D}\left[\psi\left(\frac{1}{2}\right)-\psi\left(\frac{1}{2}+\alpha\right)\right]\left\{\ln \left(\frac{1}{2 \pi T_{c} \tau}\right)+\ln ^{2}\left(\frac{1}{2 \pi T_{c} \tau}\right)\right\}
\end{aligned}
$$

From our knowledge of the calculation which includes the full screened Coulomb interaction we know that we should set $g=1 / 2$, from which it follows that $g / 4 \pi^{2} N(0) D=t$. Putting the Eqns. (13) and (12) into Eqn. (7) yields the MEF formula cited in Eqn. (何). It turns out that this approximation, although apparently reasonable, is not justified. If we plot the values of $P(\alpha)$ calculated by performing the sums directly, we find that they do not agree with Eqn. (4)). Consequently the $H_{c 2}(T)$ curves predicted by the analytic approximation and exact sum are also different.
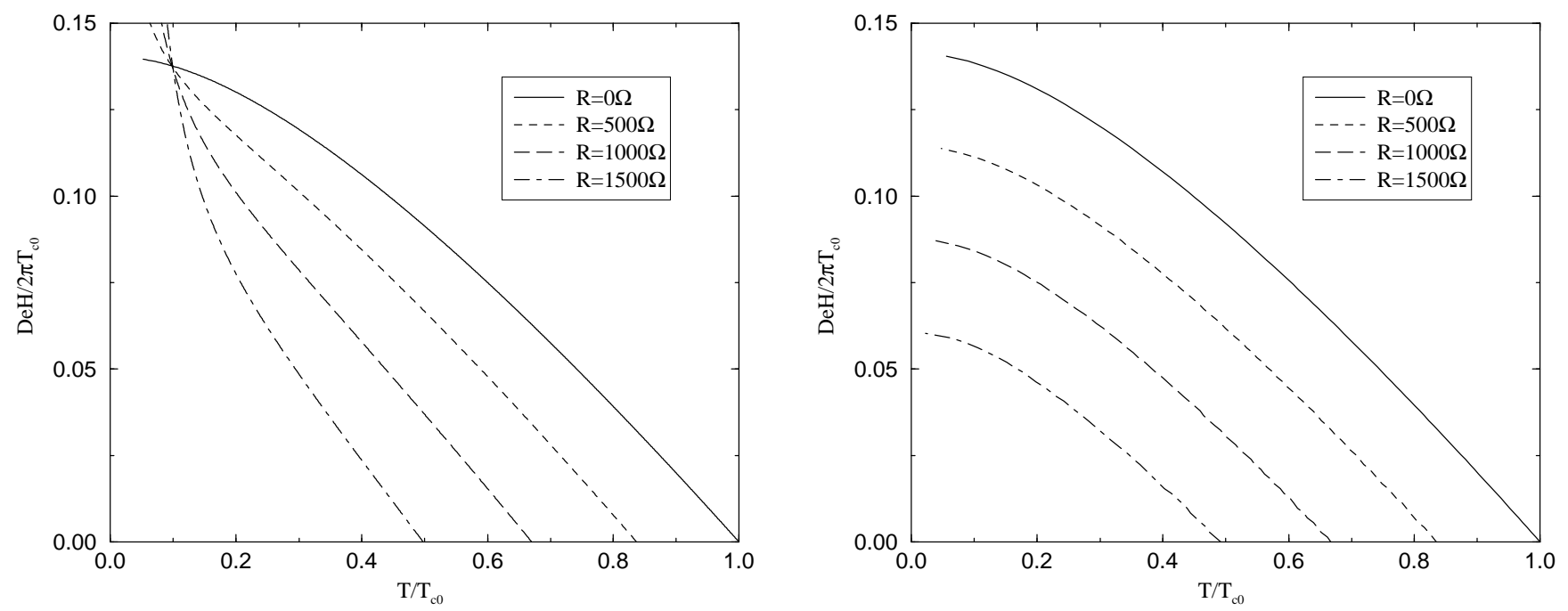

FIG. 3. Plots of $H_{c 2}(T)$ deduced from first-order perturbation theory with "self-consistency". The curves on the left are derived using the analytic MEF formula, and clearly show upward curvature at low temperature as resistance is increased. The curves on the right are derived by calculating Matsubara sums exactly, and show no upward curvature. It follows that the upward curvature is an artefact of making an incorrect analytical approximation in the derivation of the MEF formula. Both curves terminate at non-zero values of temperature because of re-entrance problems. 
On the left side of Fig. 3 we display $H_{c 2}(T)$ predicted by the MEF result of Eqn. (任) for several values of $R_{\square}$ in the case where $\ln \left(1 / 2 \pi T_{c 0} \tau\right)=6$. We clearly see the positive curvature at low temperature, and the result that the curves all terminate at finite $T$ due to re-entrance problems. On the right side of Fig. 3 we plot $H_{c 2}(T)$ deduced directly from the first-order perturbation theory result of Eqn. (11). We note that since the answers we get depend upon the logarithm of the upper cut-off, if we treat the upper cut-off differently, we will get slightly different answers. However, this difference will always be lost in fitting to experiment since we determine the unknown upper cut-off parameter from the initial slope of the data. The reason we mention this is that the exact sums can be performed in two slightly different ways: we can have the sum over $\epsilon_{l}$ and $\omega_{m}$, or the sum over $\epsilon_{l}$ and $\epsilon_{n}$, the cut-offs in each case being taken at $1 / \tau$. The results are slightly different due to different treatment of the upper cut-off. However in both cases we find no positive curvature in $H_{c 2}(T)$. There is still the problem that the curves finish at finite $T$ because of re-entrance, and this tells us that the ad hoc prescription being used to go beyond first-order perturbation theory is still inadequate. But again we stress the key point of this section: even within the ad hoc extension of first-order perturbation theory, we do not get positive curvature in $H_{c 2}(T)$ if we do the Matsubara sums exactly.

\section{OREG AND FINKELSTEIN'S RESUMMATION TECHNIQUE}

In this section we answer the question of what is the correct way to go beyond first-order perturbation theory. To do this, we extend a resummation technique recently developed by Oreg and Finkelstein 21 (OF). This involves calculating the pair scattering amplitude, $\Gamma_{c}\left(\epsilon_{n}, \epsilon_{l}\right)$, and identifying $T_{c}$ as the temperature at which it first diverges. The ladder summation involved is shown diagrammatically in Fig. 4 This is actually an extended version of the OF approach because as well as considering diagrams 3 and 4 of Fig. 2, which correspond to an effective Coulomb pseudopotential, and end up in the block $t \Lambda$, we also consider diagrams 1 and 2 of Fig. 2, which correspond to an effective self-energy, and end up in the block $\Sigma$ which renormalises the Cooperon impurity ladder. In fact we will consider both versions of the summation technique: the simpler one involves using the bare Cooperon $C_{0}$ rather than the dressed one $C$. OF demonstrate that the matrix equation they obtain in $2 \mathrm{D}$ can be approximated by a parquet-like differential equation that turns out to be identical to that obtained from renormalization group analysis. Instead of generating a continuous approximation to the matrix equation, we simply solve the equation and change the temperature (which affects the upper cut-off $M=1 / 2 \pi T \tau$ ) until we find the first zero eigenvalue of the matrix, at which point it has become singular, and we have reached $T_{c}$. Numerically this involves diagonalizing matrices of rank less than 2000 or so.

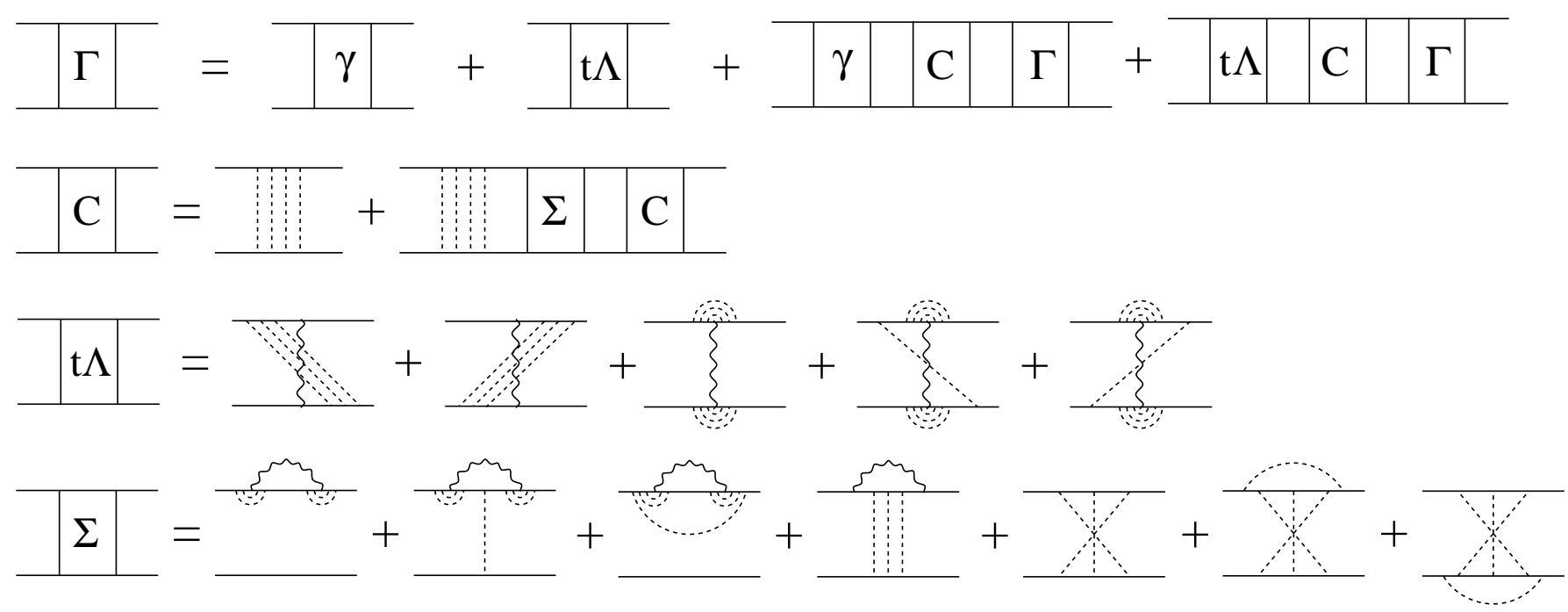

FIG. 4. Diagrammatic equation for the scattering amplitude matrix $\Gamma\left(\epsilon_{n}, \epsilon_{l}\right)$. Block $\gamma$ is the BCS interaction. Block $t \Lambda$ is the correction to the effective interaction caused by the interplay of Coulomb interaction and disorder. Block $\Sigma$ is the correction to the bare Cooperon ladder $C_{0}$ caused by Coulomb interaction and disorder. We consider both the original resummation procedure which uses the bare Cooperon $C_{0}$, and an extended procedure which includes the fully dressed Cooperon $C$.

Let us now proceed to the calculation of the pair amplitude matrix $\Gamma$. If we evaluate the diagrams of Fig. 4 , but ignore the magnetic field and self-energy correction to the Cooperon, we obtain the same equation as OF, 


$$
\Gamma\left(\epsilon_{n}, \epsilon_{l}\right)=-|\gamma|+t \Lambda\left(\epsilon_{n}, \epsilon_{l}\right)-\pi T \sum_{m=-(M+1)}^{M}\left[-|\gamma|+t \Lambda\left(\epsilon_{n}, \epsilon_{m}\right)\right] \frac{1}{\left|\epsilon_{m}\right|} \Gamma\left(\epsilon_{m}, \epsilon_{l}\right)
$$

except that we have explicitly kept both positive and negative Matsubara frequencies because the expression for $\Lambda\left(\epsilon_{l}, \epsilon_{n}\right)$ will turn out to depend upon whether its two Matsubara frequencies have the same or opposite sign. This is a reflection of the breaking of time-reversal invariance caused by the magnetic field. Let us first see how to solve the above equation, and then later put in the magnetic field, and the correction to the Cooperon. As a matrix equation, it takes the form

$$
\hat{\Gamma}=-|\gamma| \hat{1}+t \hat{\Lambda}-\frac{1}{2}[-|\gamma| \hat{1}+t \hat{\Lambda}] \hat{\epsilon}^{-1} \hat{\Gamma}
$$

where $\hat{\Gamma}_{n m}=\Gamma\left(\epsilon_{n}, \epsilon_{m}\right), \hat{1}_{n m}=1, \hat{\Lambda}_{n m}=\Lambda\left(\epsilon_{n}, \epsilon_{m}\right)$ and $\hat{\epsilon}_{n m}=(n+1 / 2) \delta_{n m}$. This has the solution

$$
\hat{\Gamma}=\hat{\epsilon}^{1 / 2}(\hat{I}-|\gamma| \hat{\Pi})^{-1} \hat{\epsilon}^{-1 / 2}(-|\gamma| \hat{1}+t \hat{\Lambda})
$$

where

$$
\hat{\Pi}=\frac{1}{2} \hat{\epsilon}^{-1 / 2}\left[\hat{1}-|\gamma|^{-1} t \hat{\Lambda}\right] \hat{\epsilon}^{-1 / 2}
$$

and $\hat{I}_{n m}=\delta_{n m}$ is the identity matrix. It follows that when the matrix $\hat{\Pi}$ has an eigenvalue equal to $1 /|\gamma|$, the pair amplitude diverges, and we have found $T_{c}$. Our approach is therefore to start at the mean field transition temperature, $T_{c 0}$, and decrease temperature until one of the eigenvalues of $(\hat{I}-|\gamma| \hat{\Pi})$ changes sign. The matrix $\hat{\Pi}(T)$ depends upon $T$ both through the dependence of $\hat{\Lambda}$ upon $T$ and through its rank $2 M$, where $M=(2 \pi T \tau)^{-1}$. We start at the mean-field value of $M$, which we will call $M_{0}$, and decrease the temperature by increasing $M$ successively by one. We diagonalize the matrix $\hat{\Pi}$ for each value of $M$ until an eigenvalue changes sign. At this point we have found $T_{c}$ for the given problem, and $T_{c} / T_{c 0}=M_{0} / M$. We can then change a parameter such as $t$ or $\alpha$ and repeat the procedure. This removes the need for any perturbative expansion.

Let us now put back in the magnetic field and Cooperon self-energy corrections. The $\left|\epsilon_{m}\right|$ denominator in the Eqn. (14) comes from the Cooperon

$$
C_{0}\left(\epsilon_{m}\right)=\frac{1}{2 \pi N(0) \tau^{2}} \frac{1}{D q^{2}+2\left|\epsilon_{m}\right|}
$$

and in the presence of a magnetic field, $\left|\epsilon_{m}\right|$ is replaced by $\left|\epsilon_{m}\right|+1 / \tau_{H}$, and hence $\hat{\epsilon}_{n m}=(n+1 / 2+\alpha) \delta_{n m}$, where $\alpha=\left(2 \pi T \tau_{H}\right)^{-1}$. The contributions to $t \Lambda$ from the diagrams of Fig. 4 which correspond to diagrams 3 and 4 of Fig. 2 are given by

$$
\begin{aligned}
& t \Lambda_{3}\left(\epsilon_{n}, \epsilon_{m}\right)=\frac{g}{\pi N(0)} \sum_{q^{\prime}} \frac{D q^{2}+D q^{2}+\left|\epsilon_{n}\right|+\left|\epsilon_{m}\right|}{\left[D q^{2}+\left|\epsilon_{n}\right|+\left|\epsilon_{m}\right|\right]} \theta\left(-\epsilon_{n} \epsilon_{m}\right) \\
& t \Lambda_{4}\left(\epsilon_{n}, \epsilon_{m}\right)=\frac{g}{\pi N(0)} \sum_{q^{\prime}} \frac{1}{\left[D q^{\prime 2}+\left|\epsilon_{n}\right|+\left|\epsilon_{m}\right|\right]} \theta\left(\epsilon_{n} \epsilon_{m}\right)
\end{aligned}
$$

and so performing the $q^{\prime}$-sum and setting $D q^{2} \rightarrow 2 / \tau_{H}$ gives

$$
t \Lambda\left(\epsilon_{n}, \epsilon_{m}\right)=\frac{g}{4 \pi^{2} N(0) D} \begin{cases}\ln \left[\frac{1}{\left(\left|\epsilon_{n}\right|+\left|\epsilon_{m}\right|\right) \tau}\right] & \epsilon_{n} \epsilon_{m}>0 \\ \ln \left[\frac{1}{\left(\left|\epsilon_{n}\right|+\left|\epsilon_{m}\right|\right) \tau}\right]+\frac{2}{\left(\left|\epsilon_{n}\right|+\left|\epsilon_{m}\right|\right) \tau_{H}} & \epsilon_{n} \epsilon_{m}<0\end{cases}
$$

It follows that the matrix elements of $\hat{\Lambda}$ are

$$
\Lambda_{n m}= \begin{cases}\ln \left(\frac{M}{n+m+1}\right) & \epsilon_{n} \epsilon_{m}>0 \\ \ln \left(\frac{M}{n+m+1}\right)+\frac{2 \alpha}{(n+m+1)} & \epsilon_{n} \epsilon_{m}<0\end{cases}
$$

To include the self-energy correction into the Cooperon, we note that the corrected Cooperon is given by 


$$
C=\left[C_{0}^{-1}-\Sigma\right]^{-1}=\frac{1}{2 \pi N(0) \tau^{2}}\left[D q^{2}+2\left|\epsilon_{m}\right|-\frac{1}{2 \pi N(0) \tau^{2}} \Sigma\right]^{-1}
$$

so that we need to absorb a factor $-1 / 2 \pi N(0) \tau^{2}$ into $\Sigma$. The contributions from the diagrams of Fig. 4 which correspond to diagrams 1 and 2 of Fig. 2 are

$$
\begin{aligned}
& \Sigma_{1}\left(\epsilon_{n}\right)=\frac{2 g}{N(0)} T \sum_{\epsilon_{m}} \sum_{q^{\prime}}\left[\frac{D q^{2}+D q^{\prime 2}+3\left|\epsilon_{n}\right|+\left|\epsilon_{m}\right|}{\left(D q^{\prime 2}+\left|\epsilon_{n}\right|+\left|\epsilon_{m}\right|\right)^{2}}\right] \theta\left(-\epsilon_{n} \epsilon_{m}\right) \\
& \Sigma_{2}\left(\epsilon_{n}\right)=-\frac{2 g}{N(0)} T \sum_{\epsilon_{m}} \sum_{q^{\prime}} \frac{1}{\left(D q^{\prime 2}+\left|\epsilon_{n}\right|+\left|\epsilon_{m}\right|\right)} \theta\left(\epsilon_{n} \epsilon_{m}\right)
\end{aligned}
$$

which partially cancel to give the result

$$
\Sigma\left(\epsilon_{n}\right)=\frac{g}{2 \pi N(0) D} T \sum_{m} \frac{1}{\left(\left|\epsilon_{n}\right|+\left|\epsilon_{m}\right|\right)} \theta\left(-\epsilon_{n} \epsilon_{m}\right)=\frac{g}{4 \pi^{2} N(0) D}\left(\sum_{k=n+1}^{M} \frac{1}{k}\right)\left[D q^{2}+2\left|\epsilon_{n}\right|\right]
$$

The weak-localization contribution to the Cooperon self-energy is given by

$$
\Sigma_{W L}\left(\epsilon_{n}\right)=-\frac{1}{2 \pi N(0)} \sum_{q^{\prime}} \frac{2 D\left(q^{2}+q^{\prime 2}\right)+4\left|\epsilon_{n}\right|}{D q^{\prime 2}+2\left|\epsilon_{n}\right|}=-\frac{1}{8 \pi^{2} N(0) D} \ln \left(\frac{1}{2\left|\epsilon_{n}\right| \tau}\right)\left[D q^{2}\right]
$$

Incorporating $\Sigma$ into the Cooperon means that $\hat{\epsilon}$ has elements

$$
\hat{\epsilon}_{n m}=\left\{\left(n+\frac{1}{2}+\alpha\right)\left[1+t \sum_{k=n+1}^{M} \frac{1}{k}\right]-2 \alpha t \ln \left(\frac{M}{n+1 / 2}\right)\right\} \delta_{n m}
$$

Using the new formulas for $\hat{\epsilon}$ and $\hat{\Lambda}$, we now plot $H_{c 2}(T)$ for various values of $t=R_{\square} / R_{0}$. The results are shown in Fig. 5: the plot on the left does not include self-energy corrections; the plot on the right does. The results are very similar and show no sign of upward curvature in $H_{c 2}(T)$. We note that there are no re-entrance problems in our calculation of $T_{c}$ : we can plot the curves down to as low a temperature as we like if we are prepared to diagonalize large enough matrices.
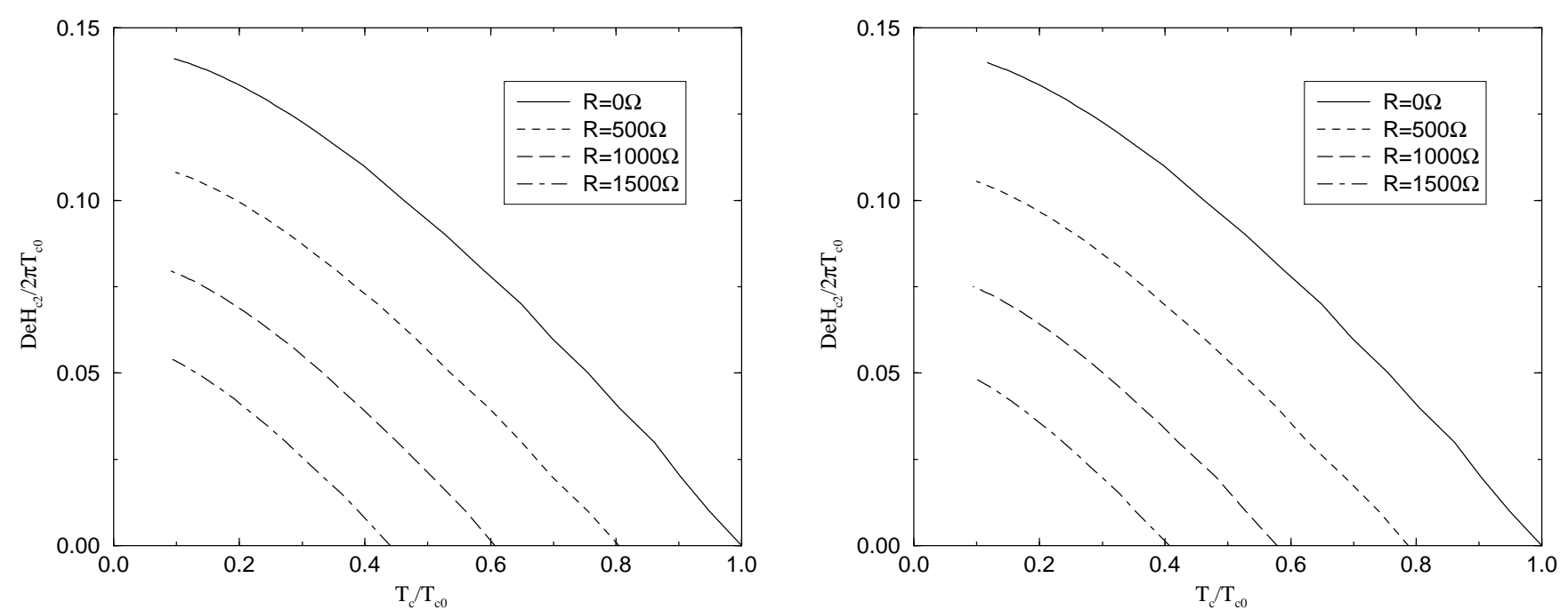

FIG. 5. Plots of $H_{c 2}(T)$ deduced from the non-perturbative resummation method. The curve on the right includes the self-energy corrections to the Cooperon ladder; the curve on the left does not. Neither curve show any sign of positive curvature at low temperature.

\section{DISCUSSION AND CONCLUSIONS}

The central message of the paper is that the theory of localization and interaction does not predict an anomalous positive curvature in the upper critical field $H_{c 2}(T)$ of thin film superconductors. A subsidiary message is that the 
resummation method developed by Oreg and Finkelstein21 is a very powerful and adaptable tool for going beyond perturbation theory in disordered superconductors. We suspected that the positive curvature in $H_{c 2}(T)$ was an artefact related to the re-entrance problem found in $T_{c}\left(R_{\mathbf{a}}\right)$, and that it would not survive a systematic non-perturbative treatment. The latter is true, but the cause of the positive curvature artefact turns out to be an incorrect analytic approximation to sums of Matsubara frequencies.

We will now consider the experimental situation by attempting to fit experimental data to both the MEF formula of Eqn. (1), and the exact first-order perturbation theory result of Eqn. (11). The data which fits best to the localization theory is that of Graybeal and Beasleyd on amorphous films of Mo-Ge, because values of $T_{c}$ for all films can be obtained by choosing a single value of the parameter $\beta=\ln \left(1 / 2 \pi T_{c 0} \tau\right)$. The fits in Fig. 6 used the parameter value $\beta=7.17$ for the MEF curves and $\beta=5.3$ for the exact sum curves. Values of $T_{c 0}=7.2 \mathrm{~K}$ and $H_{c 0}=120 \mathrm{kOe}$ were taken directly from the data. The experimental data appears to fit better to the exact sum curves than the MEF curves, and there seems little sign of an upward curvature in $H_{c 2}(T)$.

When the same procedure is applied to the data of Okuma et all5 on $\mathrm{Zn}$ films, we find that we need to use different values of $\beta$ for the two films to get the correct $T_{c}$ for given $R_{\mathrm{a}}$. The plots in Fig. 7 use values $\beta=7.5$ for the $400 \Omega$ film and $\beta=8.1$ for the $600 \Omega$ film for the MEF formula; $\beta=5.56$ for the $400 \Omega$ film and $\beta=6.1$ for the $600 \Omega$ film for the exact sum formula. Both films have the same thickness of $100 \AA$, but different resistances, and hence different diffusion constants $D$. The latter were calculated using the material parameters for $\mathrm{Zn}$ to get values of $H_{c 2}(T=0)$ for zero-resistance films of the same composition equal to $5.08 \mathrm{kOe}$ for the $400 \Omega$ film and $7.62 \mathrm{kOe}$ for $600 \Omega$ film. $T_{c 0}$ was taken to be $1 K$. Again the data seems to fit better to the exact sum curves than the MEF formula.

To fit the data of Hebard and Paalanen 14 on amorphous $\mathrm{In}-\mathrm{InO}_{x}$ films again requires a different value of $\beta$ for each film. The plots in Fig. 8 use $\beta=3.5$ for the $2250 \Omega$ film and $\beta=4.0$ for the $2900 \Omega$ and $3300 \Omega$ films for the MEF curves; $\beta=1.6$ for $2250 \Omega$ film and $\beta=2.0$ for the $2900 \Omega$ and $3300 \Omega$ films for the exact sum curves. The value of $T_{c 0}=3.6 \mathrm{~K}$ quoted in the paper was used, and a value of $H_{c 2}(0)=100 \mathrm{kOe}$ was estimated from the experimental plots. There is a clear upturn in $H_{c 2}(T)$ at low $T$, but it is not explained by the MEF curves which are effectively all upturn at these parameter values. Again the fit to exact sum curves seems better.

In conclusion it seems that the experimental curves fit better to the exact sum curves than the MEF curves. Any low temperature upturns in $H_{c 2}(T)$ are not explained by the MEF formula, and presumably correspond to different physics.

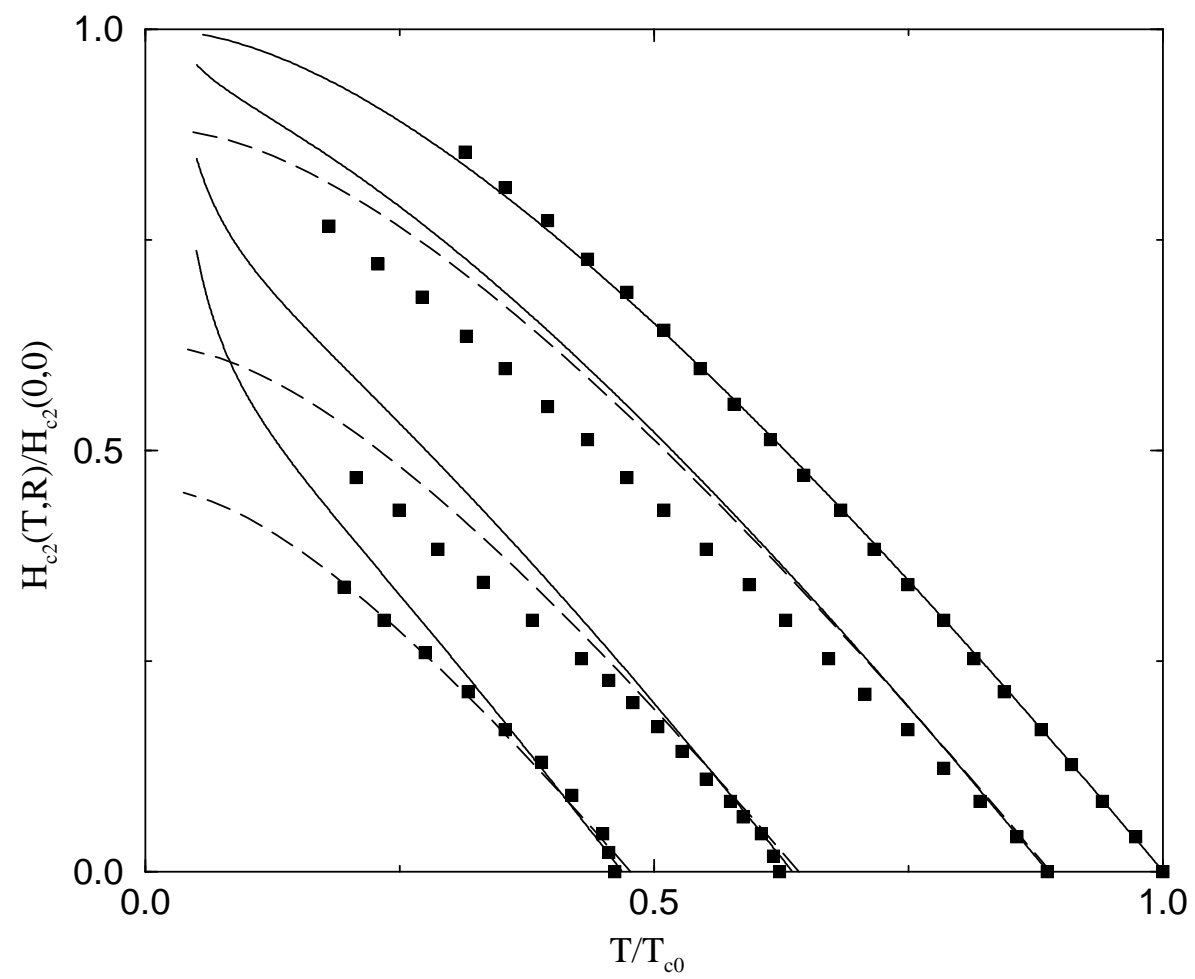

FIG. 6. Fitting of Graybeal and Beasley's data to the MEF formula (solid lines) and perturbation theory with exact sums (dashed lines). In each case only a single fitting parameter is needed for all curves, corresponding to the initial slope of the $T_{c}\left(R_{\square}\right)$ curve. The films have resistances $R_{\square} \approx 0,110,400,600 \Omega$. 


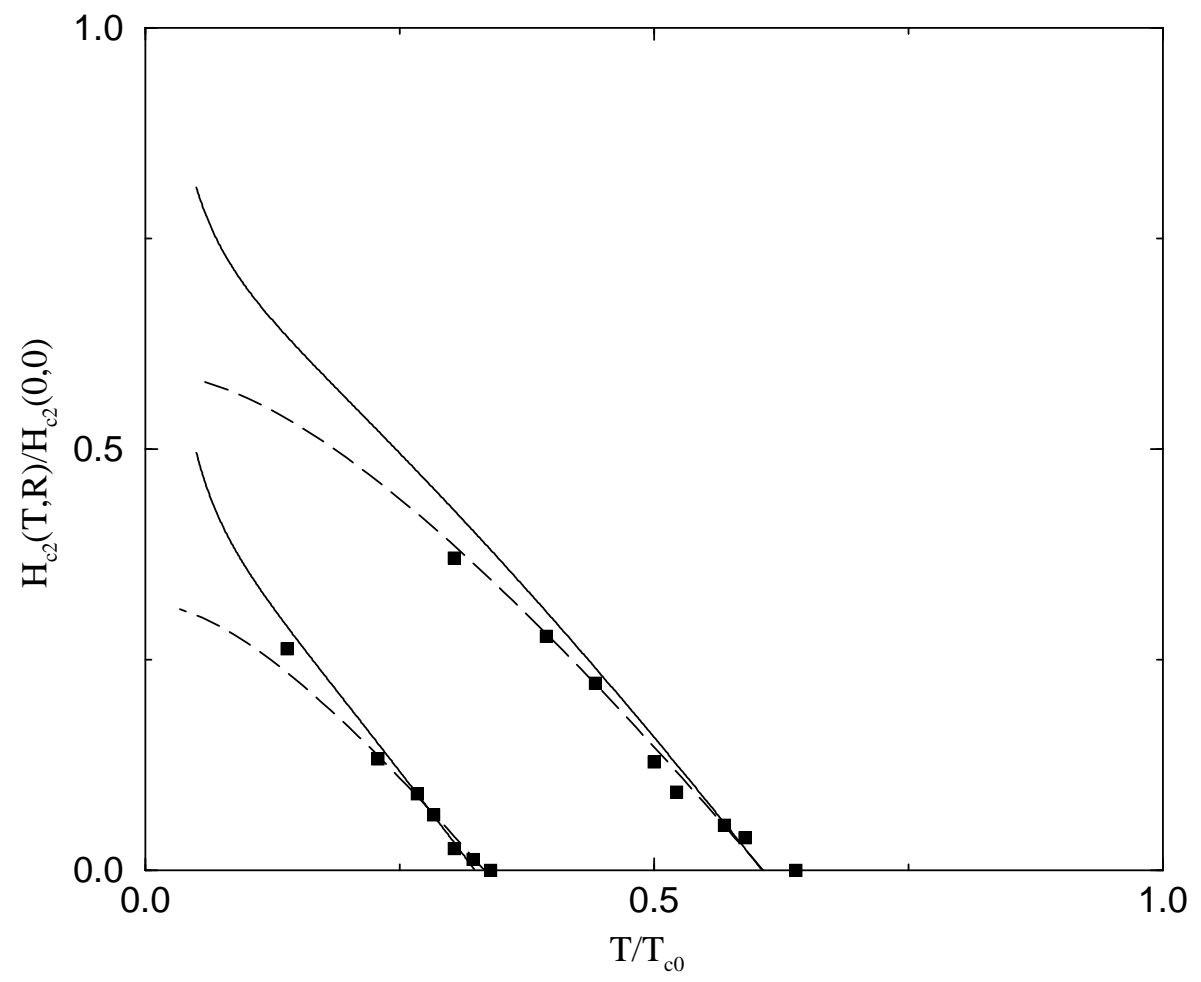

FIG. 7. Fitting of Okuma et al's data15 to the MEF formula (solid lines) and perturbation theory with exact sums (dashed lines). Different values of the fitting parameter are needed for the two films to get the correct value of $T_{c}$ for the given $R_{\square}$. The films have resistances $R_{\mathbf{\square}}=400,600 \Omega$.

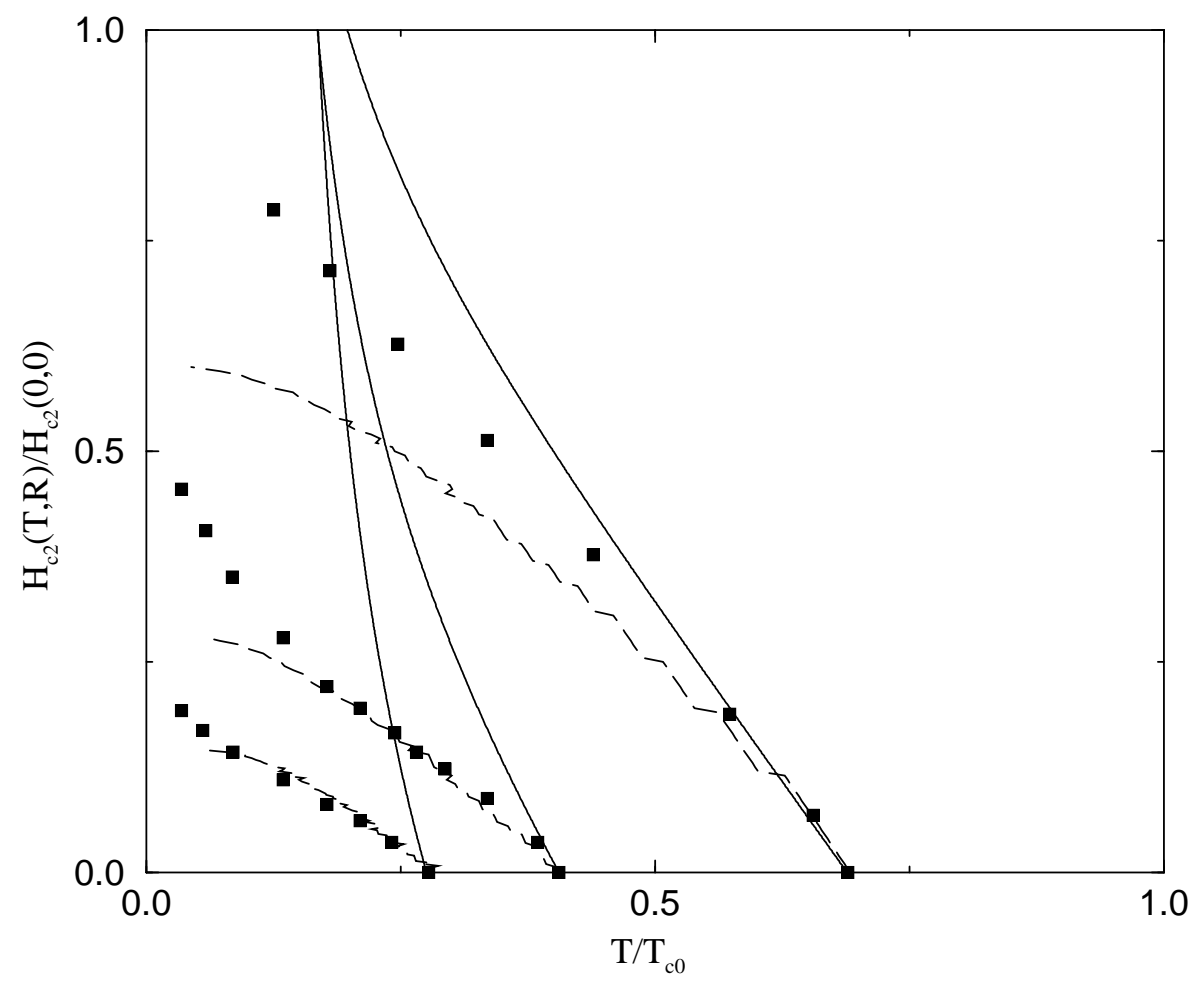

FIG. 8. Fitting of Hebard and Paalanen's data14 to the MEF formula (solid lines) and perturbation theory with exact sums (dashed lines). Different values of the fitting parameter are needed for the three films to get the correct value of $T_{c}$ for the given $R_{\square}$. The films have resistances $R_{\square}=2250,2900,3300 \Omega$. 


\section{ACKNOWLEDGEMENTS}

We thank I. Aleiner, A. Clerk, A.M. Finkel'stein, I.V. Lerner and Y. Oreg for helpful discussions. RAS acknowledges the support of a Nuffield Foundation Award to Newly Appointed Lecturers in Science and Mathematics. BSH acknowledges the support of a UK EPSRC Graduate Studentship. VA is supported by the US National Science Foundation under grant DMR-9805613.

${ }^{1}$ S. Maekawa and H. Fukuyama, J. Phys. Soc. Jpn 51, 1380 (1982).

${ }^{2}$ H. Takagi and Y. Kuroda, Solid State Commun. 41, 683 (1982).

${ }^{3}$ A.M. Finkel'stein, JETP Lett. 45, 46 (1987).

${ }^{4}$ R.A. Smith, M.Y. Reizer and J.W. Wilkins, Phys. Rev. B 51, 6470 (1995)

${ }^{5}$ A useful review of the whole area can be found in: A.M. Finkel'stein, Physica 197B, 636 (1994).

${ }^{6}$ H.R. Raffy, R.B. Laibowitz, P. Chaudhari and S. Maekawa, Phys. Rev. B 26, 6607 (1983).

${ }^{7}$ J.M. Graybeal and M.R. Beasley, Phys. Rev. B 29, 4167 (1984).

${ }^{8}$ D.B. Haviland, Y. Liu and A.M. Goldman, Phys. Rev. Lett. 62, 2180 (1989).

${ }^{9}$ S.J. Lee and J.B. Ketterson, Phys. Rev. Lett. 64, 3078 (1990)

${ }^{10}$ P. Xiong, A.V. Herzog and R.C. Dynes, Phys. Rev. Lett. 78, 927 (1997).

${ }^{11}$ J.M. Valles Jnr, R.C. Dynes and J.P. Garno, Phys. Rev. B 40, 6680 (1989); Phys. Rev. Lett. 69, 3567 (1992).

12 J.M. Valles Jnr, S-Y Hsu, R.C. Dynes and J.P. Garno, Physica 197B, 522 (1994).

13 J.A. Chervenak and J.M. Valles Jnr, Phys. Rev. B 51, 11977 (1995).

14 A.F. Hebard and M.A. Paalanen, Phys. Rev. B 30, 4063 (1984).

${ }^{15}$ S. Okuma, F. Komori, Y. Ootuka and S. Kobayashi, J. Phys. Soc. Jpn. 52, 3269 (1983).

${ }^{16}$ R.A. Smith and V. Ambegaokar, in preparation.

17 S. Maekawa, H. Ebisawa and H. Fukuyama, J. Phys. Soc. Jpn. 52, 1352 (1983).

18 A.A. Golubov and V.V. Dorin, J. Low. Temp. Phys. 78, 378 (1990).

${ }^{19}$ B. Brandow, Phys. Rep. 296, 1 (1998).

${ }^{20}$ A.M. Finkel'stein, Sov. Phys. JETP 57, 97 (1983); Z. Phys. B 56, 189 (1984).

${ }^{21}$ Y. Oreg and A.M. Finkel'stein, Phys. Rev. Lett. 83, 191 (1999).

${ }^{22}$ A.A. Abrikosov and L.P. Gor'kov, Sov. Phys. JETP 12, 1243 (1961).

23 S. Hikami, Phys. Rev. B 24, 2671 (1981). 